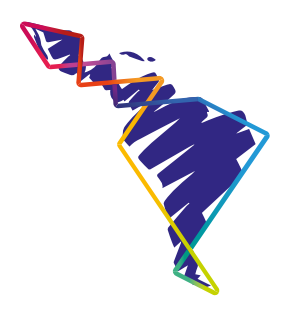

\title{
Retos que enfrenta el derecho a la educación en las zonas rurales de El Salvador
}

\author{
Challenges facing the right to \\ education in rural areas of El Salvador
}

\author{
Desafios do direito à educação nas \\ áreas rurais de El Salvador
}

Emma Patricia M uñoz Zepeda ${ }^{1}$

\begin{abstract}
Resumen
El presente texto ofrece un análisis del derecho a la educación de la niñez y adolescencia de las zonas rurales de El Salvador, como un derecho fundamental y un deber del Estado, en cumplimiento de los convenios y tratados internacionales que se han ratificado. Esta temática amerita un examen de los retos que presenta el acceso a la educación de la niñez y la adolescencia, a partir del contexto previo y posterior a la pandemia por COVID-19, la cual requirió el cambio de modalidad en el desarrollo del año lectivo. En consecuencia, se pretende exponer posibles alternativas para afrontar los desafíos identificados, a través de la aplicación de acciones positivas orientadas a su accesibilidad, presupuesto, entre otras que por verbigracia se orienten a disminuir la brecha digital existente. Se toma como parámetro que el derecho a la educación debe sustentarse en el principio de prioridad absoluta e interés superior.
\end{abstract}

Palabras clave: derecho a la educación, derecho humano, acceso a la educación, brecha digital.

\begin{abstract}
This text offers an analysis of the right to education of children and adolescents in rural areas of El Salvador, as a fundamental right and a duty of the State in compliance with ratified international conventions and treaties. This topic requires an analysis of the challenges presented by access to education for children and adolescents from the context before and after the COVID-19 pandemic, which required a change in the modality in the development of the school
\end{abstract}

Recibido: 27-6-2021 - Aceptado: 5-10-2021

1 Abogada y notaria. Abogada en el libre ejercicio. Máster en Derecho de Familia (Universidad Evangélica de El Salvador). Licenciada en Ciencias Jurídicas (Universidad Tecnológica de El Salvador). Correo electrónico: emmapatricia.zepeda@gmail.com. (D) https://orcid.org/0000-0001-5834-8876 
year. Consequently, it is intended to expose possible alternatives to face the challenges identified through the application of positive actions aimed at their accessibility, budget, among others that, for example, are aimed at reducing the existing digital divide. Taking as a parameter that the right to education must be based on the principle of absolute priority and superior interest.

Keywords: Right to education; human right; access to education; digital divide.

\section{Resumo}

Este texto oferece uma análise do direito à educação das crianças e adolescentes do meio rural de El Salvador, como direito fundamental e dever do Estado em cumprimento às convenções e tratados internacionais ratificados. Este tema requer uma análise dos desafios apresentados pelo acesso à educação de crianças e adolescentes no contexto anterior e posterior à pandemia COVID-19, o que exigiu uma mudança na modalidade no decorrer do ano letivo. Consequentemente, pretende-se expor possíveis alternativas para fazer ante os desafios identificados através da aplicação de ações positivas voltadas à sua acessibilidade, orçamento, entre outras que, por exemplo, visam reduzir a exclusão digital existente. Tomando como parâmetro que o direito à educação deve basear-se no princípio da prioridade absoluta e do interesse superior.

Palavras-chave: Direito à educação; direito humano; acesso à educação; exclusão digital.

\section{Introducción}

El derecho a la educación de las niñas, los niños y adolescentes del país se encuentra regulado con la finalidad de brindar los conocimientos, herramientas y habilidades necesarios para su desarrollo personal como profesionales. Este debe garantizarse mediante las acciones positivas que el Estado genere, de conformidad con el principio de prioridad absoluta y corresponsabilidad.

La educación es un derecho humano que encuentra su basamento en la característica de indivisible, al permitir la realización de otros derechos, a partir de su ejercicio, que conllevan el desempeño integral de cada niña, niño y adolescente, a través de una enseñanza holística que brinde las herramientas para fomentar sus habilidades; por ello, es fundamental su reconocimiento como derecho humano en igualdad de condiciones (UNESCO, 2020).

Con base en lo antes citado, es de mencionar que el derecho en cuestión es trascendental en el desarrollo de las niñas, los niños y adolescentes de las zonas rurales, lo que permitirá adquirir los conocimientos y habilidades para el cumplimiento de su plan de vida. En este período de pandemia, se ha evidenciado que la brecha digital existente permea el pleno goce de los derechos propios de la población mencionada, pues, para el 2017, se reportó, en El Salvador, que menos del $20 \%$ de 
sus domicilios estaba conectado a Internet y el acceso a planes de datos limitados no permite la descarga de archivos o videos (Venturini, 2020).

Partiendo de lo anterior, se realizó una investigación documental que ha permitido identificar elementos importantes del tema en análisis. Para ello, se consultó material bibliográfico que incluye instrumentos legales de carácter nacional e internacional, informes e investigaciones periodísticas.

En ese sentido, en la primera parte de este trabajo, se despliega el apartado "el derecho a la educación de niñas, niños y adolescentes", el cual tiene como finalidad brindar una lectura sobre la regulación normativa del derecho a la educación y las líneas de acción establecidas para su garantía y protección.

Se prosigue con la sección denominada "el derecho a la educación de la niñez y adolescencia de las zonas rurales en período de pandemia COVID-19", en la que se identifican las deficiencias educativas en el área rural de El Salvador, así como sus repercusiones en la enseñanza y el aprendizaje. Aunadas a lo anterior, se mencionan las privaciones que se manifestaron en el transcurso de los años previos a la pandemia y que aún son visibles. Luego, se concluye pon propuestas de acciones positivas por implementar, en aras de permitir el acceso a la educación de la niñez y la adolescencia de una forma integral.

\section{El derecho a la educación de las niñas, los niños y adolescentes}

De conformidad con la Constitución de la República de El Salvador, (1983), el derecho a la educación es una obligación del Estado que se regula en su artículo 53, el cual debe ser garantizado de forma integral a la niñez y adolescencia de la zona tanto urbana como rural. Asimismo, el Constituyente estableció que la educación debe tener como finalidad:

Lograr el desarrollo integral de la personalidad en su dimensión espiritual, moral y social; contribuir a la construcción de una sociedad democrática más próspera, justa y humana; inculcar el respeto a los derechos humanos y la observancia de los correspondientes deberes; combatir todo espíritu de intolerancia y de odio; conocer la realidad nacional e identificarse con los valores de la nacionalidad salvadoreña; y propiciar la unidad del pueblo centroamericano.

Es decir, la educación debe ser visualizada de un modo íntegro que permita formar a niñas, niños y adolescentes, tomando de base los derechos humanos, concebidos como piedra angular en el desarrollo de sus actividades y su propio respeto, lo cual es parte del sistema democrático que rige las acciones del Gobierno y de la sociedad. Asimismo, tal formación resulta esencial al construir la identidad, a 
través del conocimiento, de la realidad nacional, lo que posibilitará a niñas, niños y adolescentes conocer la historia de su país y comprender los acontecimientos sociales suscitados en la actualidad, a partir de los hechos, desde la perspectiva cultural, económica, social, política, entre otras.

Acorde con lo previo, la educación es concebida como un derecho para la niñez y la adolescencia, pero, a su vez, es un deber para el Estado como garante de su cumplimiento. Además, a partir de la doctrina de protección, la familia tiene un rol fundamental en la formación de sus hijas e hijos, a través de la inscripción en los centros educativos, ya sean públicos o privados, lo cual tiene su base en el Principio de rol primero y fundamental de la familia, regulado en el artículo 9 de la Ley de Protección Integral de la Niñez y la Adolescencia (2009).

La referida ley, en su artículo 81, concibe el derecho a la educación y cultura de la niñez y adolescencia en las siguientes aristas: personalidad, aptitudes y capacidades tanto mentales como físicas que permitan su desarrollo integral y las habilidades para desempeñar su rol en la sociedad. A continuación, en la tabla 1, se detallan los elementos primordiales que el legislador ha considerado para el disfrute pleno del derecho a la educación:

\section{Tabla 1}

Elementos primordiales del derecho a la educación

\begin{tabular}{|c|c|}
\hline Elemento & Contenido \\
\hline $\begin{array}{l}\text { El derecho a la educación debe } \\
\text { orientarse. }\end{array}$ & $\begin{array}{l}\text { Al pleno ejercicio de la ciudadanía; al respeto de } \\
\text { los derechos humanos; a la equidad de género; } \\
\text { al fomento de valores; al respeto de la identidad } \\
\text { cultural propia; a la paz, la democracia, la } \\
\text { solidaridad, la corresponsabilidad familiar y la } \\
\text { protección del medio ambiente. }\end{array}$ \\
\hline $\begin{array}{l}\text { El acceso a la educación } \\
\text { comprende. }\end{array}$ & $\begin{array}{l}\text { Amplia cobertura territorial en todos los niveles } \\
\text { educativos; adecuada infraestructura; idóneas } \\
\text { modalidades, planes y programas de educación; } \\
\text { docencia cualificada; suficientes recursos } \\
\text { pedagógicos, tecnológicos y espacios culturales y } \\
\text { recreativos. Además, deberá garantizar el acceso y } \\
\text { la permanencia de niñas, niños y adolescentes en el } \\
\text { sistema educativo, en condiciones de igualdad y sin } \\
\text { ningún tipo de discriminación. }\end{array}$ \\
\hline
\end{tabular}




\begin{tabular}{ll|}
\hline \multicolumn{1}{c|}{ Elemento } & \multicolumn{1}{c|}{ Contenido } \\
\hline Educación gratuita y obligatoria & $\begin{array}{l}\text { La educación inicial, parvularia, básica, media y } \\
\text { especial será gratuita y obligatoria, incluyendo los } \\
\text { centros públicos de desarrollo infantil. }\end{array}$ \\
\hline Educación y discapacidad & $\begin{array}{l}\text { Programas integrados o especiales para asegurar } \\
\text { a la niñez y la adolescencia el acceso efectivo a la } \\
\text { educación, la capacitación y las oportunidades de } \\
\text { esparcimiento. }\end{array}$ \\
\hline
\end{tabular}

Nota. Elaboración propia, con base en la Ley de Protección Integral de la Niñez y la Adolescencia (2009). Artículos 81, 82 y 83.

Partiendo de lo antes mencionado, el derecho a la educación es fragmento importante en el desarrollo de todo ser humano, pues fortalece y brinda las bases para el proyecto de vida de cada niña, niño y adolescente; en consecuencia, repercute en la sociedad y en el capital humano que se requiere para el desenvolvimiento de las naciones. Es por ello que el Comité de Derechos Económicos, Sociales y Culturales establece un rol preponderante a la formación, al concebirla como el "principal medio que permite a adultos y menores marginados económica y socialmente salir de la pobreza y participar plenamente en sus comunidades" (Comité sobre los Derechos del Niño [CDN], 1999, párr. 1).

Es decir, el derecho educativo debe ser accesible a todas y todos; para ello, el Estado debe crear los mecanismos legales, institucionales y presupuestarios que vuelvan factible esta característica. Tanto es así que el Sistema Nacional de Protección Integral de la Niñez y la Adolescencia, como organismo coordinado de instituciones que deben garantizar los derechos de este sector de la población y en especial referencia el derecho a la educación, establece, en la Política Nacional de Protección Integral de la Niñez y de la Adolescencia, como parte de sus directrices, específicamente en el tema en análisis, en su estrategia 3.5, de su objetivo estratégico 3, que se debe "garantizar calidad de educación y en igualdad de condiciones para todas las niñas, niños y adolescentes".

\section{EI derecho a la educación de la niñez y adolescencia de las zonas rurales en período de pandemia COVID-19}

A partir de la declaratoria de emergencia por la pandemia de COVID-19 en El Salvador, los centros educativos públicos y privados fueron cerrados, con la finalidad de evitar contagios masivos, así como de proteger la salud de la niñez y la adolescencia. El Estado, como garante del derecho a la educación, estableció mecanismos para continuar con el año escolar y evitar que niñas, niños y adolescentes perdieran su año lectivo. En este sentido, en la tabla 2, se detalla las medidas adoptadas por el ente estatal: 


\section{Tabla 2}

Medidas adoptadas por el Gobierno para garantizar el derecho a la educación

\section{Período}

Contención de la emergencia (del 14 de marzo al 14 de abril)
Acciones

Elaboración, producción y distribución de materiales y guías educativas para apoyar la labor docente.

Se giraron orientaciones para docentes y estudiantes.

Se creó el micrositio www.mined.gob.sv/

emergenciacovid19, para alojar contenido académico y orientaciones a la comunidad educativa, el cual ha tenido más de 4000000 de visitas.

Se habilitó un "call center" (2592-2009 y 60083286) para atender a la comunidad educativa, que superó las 7000 consultas, denuncias y orientaciones pedagógicas.

Priorización del Currículo Nacional, mejoras en la distribución de materiales e introducción de nuevas plataformas.

Se distribuyeron guías impresas para más de 200000

Integración de diversas plataformas (del 15 de abril al 24 de mayo) estudiantes con mayores dificultades para acceder a Internet.

Se inició la formación docente, junto con la Secretaría de Innovación de la Presidencia, en el uso de la plataforma de Google Classroom. Más de 30 000 docentes y asistentes técnicos comenzaron su capacitación.

Lanzamiento de la franja de televisión educativa "Aprendamos en Casa".

Digitalización de la educación (del 25 de mayo en adelante)

Lanzamiento del programa radial "Crecer Leyendo". Distribución de guías impresas para más de 200000 estudiantes.

Habilitación del contenido digital en Google Classroom.

Se cuenta con un plan para el retorno a la

El retorno a la escuela (la fecha se establecerá a partir de las directrices del MINSAL) multiplataforma, a fin de garantizar la continuidad educativa del estudiantado salvadoreño.

Nota. Elaboración propia, a partir de datos de la Memoria de Labores 2019-2020. (MINED, 2020).

Las acciones enunciadas responden a las responsabilidades adquiridas por el Estado en la garantía del derecho a la educación de la niñez y la adolescencia; no obstante, el alcance de estas no ha permeado de la manera que se vislumbraba, específicamente 
en la zona rural. Cabe señalar los siguientes elementos: no se cuenta con dispositivos de comunicación; se presenta la falta de recursos económicos para realizar recarga de saldo para el uso de Internet; madres y padres de familia no cuentan con un nivel educativo que les permita apoyar a sus hijas e hijos en la elaboración de las tareas o en dudas que se les puedan presentar; existen limitaciones en las herramientas o materiales necesarios para el desarrollo de sus actividades, de acuerdo con los requerimientos de las guías académicas (Moreno, 2021).

Según un informe de la Fundación para la Educación Superior, se ha identificado en el área urbana un mayor índice de cobertura, en comparación con la zona rural de país. En esta última, hay una mayor incidencia de la radio, además, se identificó que el acceso a computadora e Internet en las viviendas no es mayor al $5 \%$. Para el 2019, se determinó que solo el $19.6 \%$ de la población habitante de esos lugares tiene facilidad para el uso de una computadora personal y un $8.2 \%$ utiliza Internet en horas semanales. Tales datos representan que niñas, niños y adolescentes de esa zona del país se encuentran en menos contacto con este tipo de dispositivos de comunicación (FES, 2020).

La realidad en la zona rural presenta sus particularidades desde las apreciaciones de las madres y padres de familia; la pandemia por COVID-19 ha representado un reto en el nivel educativo. De acuerdo con un reportaje periodístico, se identifica que «las mamás y los papás dicen que los docentes "ya no están haciendo nada" y hoy les toca todo a ellas y ellos. Opinan que los docentes creen que no tienen nada más que hacer y que pueden asumir los costos inesperados de internet y saldo para el teléfono» (Martín, 2020).

Con el apoyo de la cita, se puede mencionar el caso del Centro Escolar caserío San Cristóbal, el cual está ubicado en la zona rural del municipio de Guazapa, departamento de San Salvador. Allí, se identifica, como resultado de esta pandemia, los siguientes retos: alta de conectividad; poca motivación de algunos padres de familia; las condiciones de vida del estudiantado; el perfil económico de las familias situado en escasos recursos; que la transición entre lo presencial y lo virtual se volvió un choque educativo, lo que produjo que estudiantes dejaran sus estudios y decidieran comenzar su trayecto laboral (Villeda, 2021).

En cuanto al acceso a Internet, el estudio denominado "Conectividad rural en América Latina y el Caribe un puente al desarrollo sostenible en tiempos de pandemia", refleja que el $90 \%$ de la población en la zona rural de El Salvador no cuenta con acceso a la red, situación que ubica al país en el clúster de baja conectividad significativa rural. Esto permitió concluir que el 71 y $89 \%$ de la ciudadanía rural 
de los países analizados, incluyendo el nuestro, no accede a servicios de conexión de calidad (IICA/ BID/MICROSOFT, 2020).

Por su parte, un estudio realizado por Oscar Picardo Joao (2005), señala que, en El Salvador, se reportan 6001 centros educativos en los niveles de parvularia, básica y media, los cuales, a su vez, se clasifican en 5015 instituciones públicas y 986 privadas. De todas ellas, un 91.83 \%, en el ámbito nacional, no posee Internet, únicamente un $5.78 \%$, el cual utiliza para fines educativos, y un $3.52 \%$ cuenta con acceso a la red, pero no la utiliza para propósitos educativos.

Asimismo, Picardo (2005) agrega que estos datos tienen incidencia en el uso del Internet en los centros educativos, pero, es de señalar que, para el período de la investigación citada en líneas previas, se visualiza una diferencia en cuanto al número de computadoras en las instituciones, de acuerdo con la población estudiantil activa, es así, que en el sector público se registraron 3.49 equipos computacionales por institución, es decir, 82.84 estudiantes por computadora. Por su parte, el sector privado reportaba 2.85 computadoras por institución, lo que representaba 104.01 estudiantes por máquina.

En este mismo orden de ideas, se registró, para el 2019, que de niñas y niños desde los cuatro años en adelante, quienes asisten a un centro educativo en El Salvador, un total de 1346384 (entre niñas, niños y adolescentes) tuvieron acceso a un centro de cómputo y 299319 de ellas y ellos no (DIGESTYC, 2020).

El escenario antes planteado, en un primer término, soslaya la desigualdad existente en el acceso al servicio de Internet que permite el ejercicio de los derechos y la construcción del conocimiento de la niñez y adolescencia. En segundo lugar, el panorama presenta desafíos en cuanto al acercamiento a los demás servicios, a través de la tecnología, al impedir comunicarse con otras personas; acceder a información mediante dispositivos digitales; adquirir conocimientos en robótica, inteligencia artificial, realidad virtual, entre otros.

Lo antes citado representa una desventaja tanto en el desarrollo como en el aprendizaje de la niñez y adolescencia; en consecuencia, estos quedan relegados y configuran una grave vulneración a los derechos humanos. Pues bien, la Asamblea General de las Naciones Unidas, en el 2011, ha declarado el acceso a Internet como un derecho humano que debe ser garantizado por los Estados. A partir de este reconocimiento, se establece lo siguiente:

Los Estados tienen la obligación de promover el acceso universal a Internet para garantizar el disfrute efectivo del derecho a la libertad de expresión. El acceso a 
Internet también es necesario para asegurar el respeto de otros derechos, como el derecho a la educación, la atención de la salud y el trabajo, el derecho de reunión y asociación, y el derecho a elecciones libres (OEA, 2011).

Efectivamente, el derecho a acceder Internet es crucial para el ejercicio del derecho a la educación de la niñez y adolescencia, en particular, en este período de pandemia, en el cual se ha declarado el cierre de las escuelas. A pesar de ello, la brecha digital requiere una praxis activa por parte del Estado, a través de la inversión; se considera que su impacto debe reflejarse en la asignación presupuestaria para el ramo de educación que considere invertir en infraestructura y equipo para las escuelas de la zona rural. Además, se precisa del asocie público-privado, en aras de establecer las coordinaciones necesarias para aproximarse al derecho en cuestión.

Aunado a lo anterior, debe incorporarse la inversión para poder ingresar a Internet en los centros educativos. Esto permitiría el uso de las plataformas digitales por parte de la planta docente y el alumnado. De igual manera, es importante la capacitación de los maestros, tomando en consideración las particularidades de sus lugares de trabajo, pues el profesorado de las zonas urbanas puede presentar otras falencias, en comparación al personal docente de las zonas rurales.

La idea antes planteada encuentra su base en lo señalado por el Comité sobre los Derechos del Niño, que establece la obligación de los Estados de realizar asignaciones presupuestarias orientadas a la creación de normativa, políticas y programas, los cuales promuevan el acceso de niñas, niños y adolescentes tanto a los entornos digitales como a la inclusión en estos, sin ningún tipo de discriminación (Comité sobre los Derechos del Niño [CDN], 2021).

Todo lo dicho se expone desde la visión de la doctrina de protección integral, que reconoce a la niñez y adolescencia como sujetos de derechos, los cuales deben ser garantizados a partir de su reconocimiento en la legislación interna, la creación de mecanismos idóneos motivadores de su ejercicio e implementación en el nivel nacional, de conformidad con el artículo 28 de la Convención sobre los Derechos del Niño. Esta última estipula el carácter primordial de los derechos comentados para la construcción del proyecto de vida de cada niña, niño y adolescente.

Es a partir de lo antes mencionado que la Ley de Protección Integral de la Niñez y la Adolescencia (2009) concibe la educación como parte de los derechos de desarrollo; en efecto, brindará las herramientas intelectuales, morales y espirituales para formar una ciudadanía respetuosa de los derechos humanos y de su nación. 
En otro orden de ideas, la realidad de la educación en la zona rural presenta desafíos necesarios de analizar, pues repercuten en el disfrute pleno de aquella por parte de la niñez y adolescencia. Esos retos se detallan a continuación:

\section{Tabla 3}

La educación en la zona rural

\section{Obstáculos identificados en la zona rural}

Para Google Classroom, el alumnado

Poca señal de celular, lo que impide enviar necesita tener una computadora. Aunque las tareas por WhatsApp. la tuvieran, tampoco se podría conectar, porque no tiene Internet en sus casas.

La o el docente debe transcribir las guías de estudio y adecuarlas al contexto de su alumnado.

Una misma o un mismo docente tiene tres secciones integradas: cuarto, quinto y sexto grado, en la misma aula y al mismo tiempo.

La falta de un sistema de actualización docente que responda a las necesidades concretas de cada maestro.

Nota. Elaboración propia, a partir de datos de Educar sin recursos y sin acompañamiento (Rosales, 2020).

A partir de lo antes citado, se puede corroborar que las zonas rurales presentan sus particularidades y requieren un análisis exhaustivo, al momento de la construcción de los contenidos, guías de trabajo, la aplicación de plataformas digitales, entre otras. Es trascendental que se retomen estas condiciones, incluyendo las experiencias de las y los docentes que viven día tras día con su estudiantado en este escenario; así podrán aportar en la mejora continua de la educación.

El Estado ha adquirido el compromiso de establecer los mecanismos y recursos necesarios para el mejor desarrollo de las habilidades de la niñez y la adolescencia, a través de la educación, lo que implica acciones positivas como las señaladas en líneas anteriores, las cuales produzcan un impacto en su ejercicio eficaz. Además, los Gobiernos deben cumplir, mediante sus políticas o programas institucionales, las siguientes características, como parte de las bases esenciales de tal derecho humano, que se detallan a continuación: 


\section{Tabla 4}

Características del derecho a la educación

\begin{tabular}{|c|c|}
\hline Características & Contenido \\
\hline Disponibilidad & $\begin{array}{l}\text { Debe haber instituciones y programas de enseñanza, en } \\
\text { cantidad suficiente, en el ámbito del Estado parte. }\end{array}$ \\
\hline Accesibilidad & $\begin{array}{l}\text { Las instituciones y los programas de enseñanza han de ser } \\
\text { accesibles para todas y todos, sin discriminación, en el ámbito } \\
\text { del Estado parte. Esto en sus tres dimensiones: } \\
\text { A) No discriminación. La educación debe ser accesible para } \\
\text { todas y todos, especialmente para los grupos no vulnerables } \\
\text { de hecho y de derecho, sin discriminación por ninguno de los } \\
\text { motivos prohibidos. } \\
\text { B) Accesibilidad material. La educación ha de ser asequible } \\
\text { materialmente, ya sea por su localización geográfica de } \\
\text { acceso razonable (por ejemplo, una escuela vecinal) o por } \\
\text { medio de la tecnología moderna (mediante el acceso a } \\
\text { programas de educación a distancia). } \\
\text { C) Accesibilidad económica. La educación ha de estar al } \\
\text { alcance de todas y todos. }\end{array}$ \\
\hline Aceptabilidad & $\begin{array}{l}\text { La forma y el fondo de la educación, comprendidos los } \\
\text { programas de estudio y los métodos pedagógicos, han de } \\
\text { ser aceptables para los estudiantes y, cuando proceda, a los } \\
\text { padres. }\end{array}$ \\
\hline Adaptabilidad & $\begin{array}{l}\text { La educación ha de tener la flexibilidad necesaria para } \\
\text { adaptarse a los requerimientos de sociedades y comunidades } \\
\text { en transformación, así como para responder a las necesidades } \\
\text { del alumnado en contextos culturales y sociales variados. }\end{array}$ \\
\hline
\end{tabular}

Nota. Elaboración propia, a partir de datos de Observación General número 13, El derecho a la educación (artículo 13 del Pacto). (Comité de Derechos Económicos, Sociales y Culturales, 1999, párr. 6).

Las características antes citadas son indispensables para la realización del derecho educativo y su influencia en el desarrollo tanto de la niñez como de la adolescencia salvadoreña, con miras a permitir la adquisición de conocimientos y herramientas orientados al crecimiento personal, intelectual y profesional de dicha población infantil y juvenil. Es de enfatizar que se presentan óbices para el pleno ejercicio de la educación, pues la realidad de las escuelas rurales difiere de lo que se establece en la tabla 4.

Puesto que las escuelas rurales son base para la enseñanza-aprendizaje de la niñez y adolescencia, de los 5147 centros educativos registrados en el país, el $74.88 \%$ se encuentra en la zona rural y solo un $25.12 \%$ en el área urbana (MINED, 2018). 
Las instituciones de la zona rural reciben, por parte del Ministerio de Educación (entidad rectora en este ramo en la asignación de presupuesto), dinero destinado a papelería, refrigerios y ordenanza. La papelería es fundamental para el desarrollo de las actividades del alumnado, pues, como se ha señalado, los materiales son escasos por las condiciones de acceso y por no contar con librerías cercanas en los caseríos o cantones, lo cual dificulta la realización de algunas actividades (Mejía, 2020).

Asimismo, se ha identificado la pobreza extrema como una limitante para el acceso a la educación, lo que implica dejar los estudios para dedicarse a labores agrícolas o la búsqueda de un trabajo que permita contribuir con las necesidades del hogar. Este punto en concreto tiene mucha relación con el trabajo infantil, el cual debe ser abordado de forma integral. También resultó un inconveniente el acceso a los centros educativos, porque estos se encuentran a largas distancias de los hogares de la niñez y la adolescencia; ello requiere que infantes y jóvenes deban desplazarse mucho. Además, las escuelas únicamente cubren hasta un grado específico y se hallan docentes que presentan niveles de preparación hasta los tres años de carrera (Araujo, 2018).

En este sentido, es insoslayable considerar las condiciones particulares de las escuelas rurales, las cuales deben ser valoradas e incluidas al momento de construir el currículum escolar. El presupuesto designado para las mejoras de los centros educativos y equipamiento permitirá disminuir la brecha que pueda existir entre estudiantes de la zona urbana y de la rural. Se busca que todas esas instituciones visualicen las características de accesibilidad, aceptabilidad y adaptabilidad, hecho que posibilitará establecer las bases de una educación integral, asequible y con las competencias necesarias para la formación de la niñez y la adolescencia.

Es así como el Comité sobre los Derechos del Niño considera relevante la asignación de recursos para el cumplimiento de los derechos sociales como la educación e invita a los Estados a hacer uso de todos los instrumentos económicos que se posean, con la finalidad de garantizar los derechos de la niñez y adolescencia. Estos gremios deben ser el centro de las decisiones y acciones por implementar en materia presupuestaria, a fin de incidir de forma favorable en su desarrollo integral (Comité sobre los Derechos del Niño [CDN], 2016, párr. 14). 


\section{Conclusión}

La pandemia por COVID-19 nos ha permitido desarrollar la educación en un contexto diferente, al trasladar la formación tradicional a nuevas maneras comunicación y retomar las existentes, con el propósito de garantizar el derecho en mención, pero vinculando la conectividad como parte fundamental en el desarrollo de las capacidades y habilidades tanto de la niñez como de la adolescencia.

La educación por impartir a la niñez y adolescencia de la zona rural debe ser analizada de forma estructural, visibilizando las deficiencias, amenazas, oportunidades y fortalezas que presenta. Esto, con el objetivo de alcanzar un mejor impacto, el cual merme el número de niñas, niños y adolescentes que abandonan sus estudios para dedicarse al trabajo infantil, tomando en consideración las circunstancias económicas de las familias y los apoyos que requieren.

Pues bien, el derecho humano de conectividad no debe soslayarse en materia de educación, dado que permite el acceso a conocimientos, información y herramientas, los cuales posibilitan a niñas, niños y adolescentes desarrollar habilidades para la vida. Todo ello representa retos para el Estado, que se deben permear en las decisiones, a fin de asegurarse este derecho, tomando como parámetro las situaciones particulares mencionadas en el presente artículo.

Asimismo, debe analizarse la relación del derecho a la educación con el derecho a la conectividad, a la luz de la doctrina de protección integral y bajo la premisa del principio de prioridad absoluta. La consecución de acciones estatales y acuerdos con instituciones privadas, en la mejora de las condiciones en infraestructura, equipamiento (pupitres, material didáctico, papelería, computadoras), acceso a Internet, personal docente capacitado y otras herramientas necesarias para brindar una educación de calidad, generará el desarrollo de habilidades competitivas; estos son eslabones que garantizan un acceso educativo gratuito y con protección de datos. 


\section{Referencias}

Araujo J. (2018). ¿Qué es una escuela rural en El Salvador? Fundación para la Educación Superior. https://www.fes.edu.sv/que-es-una-escuela-rural-en-el-salvador/

Comité de Derechos Económicos, Sociales y Culturales. (1999). Observación General 13, El derecho a la educación (artículo 13 del Pacto), párrafo 1, Ginebra.

Comité sobre los Derechos del Niño. (2021). Observación General número 25 relativa a los derechos de los niños en relación con el entorno digital, Ginebra.

Comité sobre los Derechos del Niño. (2016). Observación General número 19 (2016) sobre la elaboración de presupuestos públicos para hacer efectivos los derechos del niño (artículo 4), Ginebra.

DIGESTYC. (2020). Encuesta de Hogares y Propósitos Múltiples 2019, El Salvador. ex.php/ novedades/avisos/965-ya-se-encuentra-disponible -la-encuesta-de-hogares-de-propositos-multiples-2019.html

Fundación para la Educación Superior. (2020) ¿Aprender sin escuelas? Los desafíos de la continuidad educativa para los más vulnerables. Santa Tecla, El Salvador.

IICA/ BID/ MICROSOFT. (2020). Conectividad rural en América Latina y el Caribe un puente al desarrollo sostenible en tiempos de pandemia. https://repositorio.iica.int/ handle/11324/12896

Martín, P. (2020). La relación familia-escuela no está resistiendo la cuarentena. El Faro. net https://elfaro.net/es/202005/columnas/24387/La-relaci\%C3\%B3n-familia-escuela-no-est $\%$ C3\%A1-resistiendo-la-cuarentena.htm

Mejía, D. (2020). Investigación ECC: ¿Está lista la educación rural salvadoreña para asumir los retos de la digitalización? Conéctate con la Realidad. https://tuespacioujmd. com/2020/10/20/investigacion-ecc-esta-lista-la-educacion-rural-salvadorena-para-sumir-los-retos-de-la-digitalizacion/

Ministerio de Educación. (2020). Memoria de Labores 2019-2020. https://www.mined. gob.sv/descargas/memoria-de-labores-2019-2020.html

Ministerio de Educación. (2018). Observatorio MINED 2018 sobre los centros educativos públicos y privados subvencionados de El Salvador. https://fdocuments.ec/document/ observatorio-mined-2018-2019-02-04-cantidad-de-ce-5164-cantidad-de-docentes.html

Moreno, M. (2021). Educación en pandemia: necesidad de autonomía y formación docente. Noticias UCA. https://noticias.uca.edu.sv/conciencia-universitaria/ educacion-en-pandemia-necesidad-de-autonomia-y-formacion-docente 
OEA. (2011). Declaración conjunta sobre libertad de expresión e internet. OEA. https:// www.oas.org/es/cidh/expresion/showarticle.asp?artID $=848$

Picardo, O. (2005). Brecha digital en el sector educativo salvadoreño: retos y estrategias. Revista de Educación y Desarrollo, 4. https://www.cucs.udg.mx/revistas/edu_desarrollo/ anteriores/4/004_Picardo.pdf

Rosales, D. (2020). Educar sin recursos y sin acompañamiento. La Prensa Gráfica. Séptimo Sentido. https://7s.laprensagrafica.com/educar-sin-recursos-y-sin-acompanamiento/

UNESCO. (2020). Lo que necesita saber sobre el derecho a la educación. https://es.unesco. org/news/lo-que-necesita-saber-derecho-educacion

Venturini, J. (2020). Brecha digital y acceso. Los límites de la educación a distancia en América Latina. Derechos Digitales. Derechos Humanos y Tecnología en América Latina. https://www.derechosdigitales.org/14324/ los-limites-de-la-educacion-a-distancia-en-america-latina/

Villeda, J. (2021). El covid-19 golpea bajo a las escuelas rurales del país. Periódico El Mundo. https://diario.elmundo.sv/el-covid-19-golpea-bajo-a-las-escuelas-rurales-del-pais/ 
